\title{
DESIGN OF A NEW CAVITATION TUNNEL AT BRNO UNIVERSITY OF TECHNOLOGY FOR FSI TESTING
}

\author{
P. Čupr*, M. Cardoso*** P. Rudolf ${ }^{* * *}$
}

\begin{abstract}
The aim of this paper is to present a new cavitation tunnel, which has been designed and later will be built at Viktor Kaplan Department of Fluid Engineering at Brno University of Technology. The tunnel will be used to investigate the effect of hydrofoil trailing edge shape on wake dynamics and its interaction with the mechanical structure. Preliminary CFD tests considering $2 D$ hydrofoils were carried out to determine the vortex shedding frequency for three different trailing edge shapes. The natural frequencies of these hydrofoils were calculated to ensure, that it will be possible to observe vortex shedding in lock-in regime.
\end{abstract}

Keywords: cavitation tunnel, Karman vortex shedding, modal analysis, trailing edge

\section{Introduction}

During the operation time of the turbine runner, the excitation which is coming from the fluid can rapidly decrease the runner life time and can cause a damage of the whole runner. One of the main excitation sources is the interaction of vortex structures behind the trailing edge of the runner blades. This phenomenon, known as Karman vortex shedding, leads to periodic lift force which is acting on the blade. The frequency of the lift force can be calculated using dimensionless Strouhal number given by

$$
\mathrm{St}=\frac{f \cdot L}{U}
$$

where $f$ is frequency of vortex shedding, $L$ is characteristic length (thickness of the trailing edge) and $U$ is flow velocity. The vortex shedding can be observed if the Strouhal number is equal to its critical value around 0,2 . Then the vortex shedding frequency can be calculated. If this frequency of the excitation is near the natural frequency of the blade, the resonance may occur and can cause a damage of the whole runner (Dörfler 2013, Lockey et al., 2006). This must be prevented during the design process.

To better understand the influence of trailing edge shape on behaviour of the vortex structures, the cavitation tunnel has been designed and will be built at Viktor Kaplan Department of Fluid Engineering at Brno University of Technology. The cavitation tunnel is designed as a closed circuit (Figure 1) with two pumps which provide maximal flow rate $500 \mathrm{l} / \mathrm{s}$. The test section is $760 \mathrm{~mm}$ long and has a rectangular cross-section with dimensions $150 \times 100 \mathrm{~mm}$. The maximal flow velocity in the test section is $33 \mathrm{~m} / \mathrm{s}$. Downstream of the test section, a $7^{\circ}$-full angle diffuser with rectangular cross-section is mounted and followed by a water tank. The circuit is closed by pipes with circular cross-section of diameter $300 \mathrm{~mm}$. Upstream of the test section, a contraction of length $516 \mathrm{~mm}$ is installed, which was designed using the approach described in (Nedyalkov, 2012). Its cross-section is changing from the circular inlet of diameter $300 \mathrm{~mm}$ to the rectangular outlet. The circuit is connected to a vacuum pump to control level of static pressure in the circuit.

\footnotetext{
Ing. Pavel Čupr: Energy Institute, Brno University of Technology; Technická 2896/2; 616 69, Brno; CZ, cupr@fme.vutbr.cz

** Mateus da Silva Cardoso: Universidade Federal de Santa Catarina, Brazil; Campus Universitário Reitor João David Ferreira Lima Trindade; CEP: 88040-900, Florianópolis; BR, mateus-cardoso@outlook.com

*** Assoc. Prof. Pavel Rudolf, PhD.: Energy Institute, Brno University of Technology; Technická 2896/2; 616 69, Brno; CZ, rudolf@fme.vutbr.cz
} 
The effects of changing the trailing edge shapes on behaviour of the vortex structures will be studied using three similar hydrofoils made of steel with various trailing edge shapes, i.e. the blunt, the rounded and the $30^{\circ}$ oblique truncated trailing edge (Figure 2).

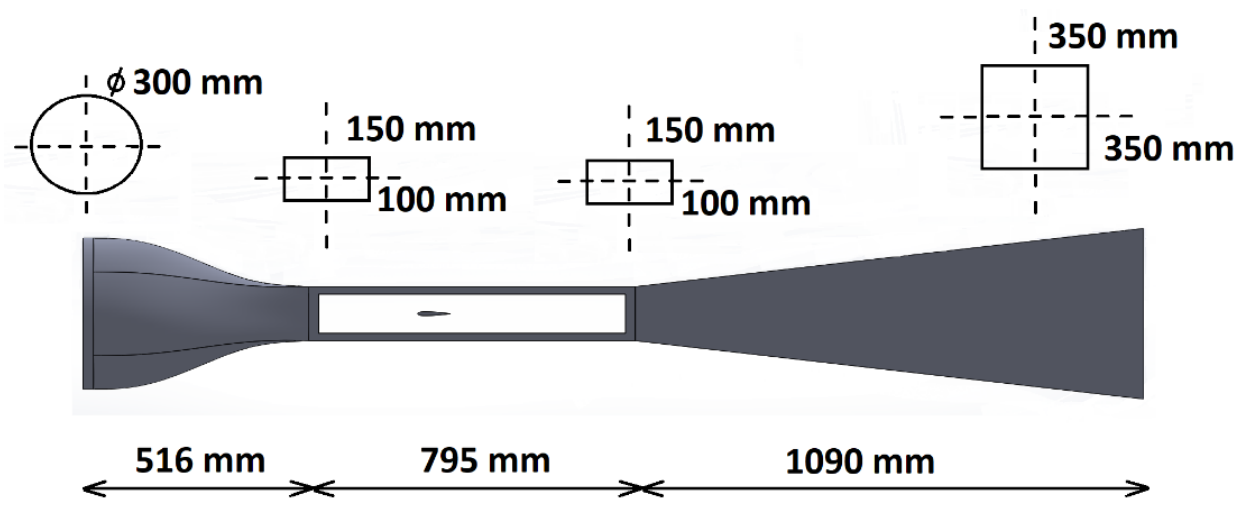

Fig. 1: Dimensions of the cavitation tunnel

\section{CFD Transient preliminary tests}

As a part of the design procedure, the transient CFD analysis of the flow around hydrofoils with three various trailing edge shapes were carried out to determine the vortex shedding frequency. Three values of flow velocity were chosen $(2 \mathrm{~m} / \mathrm{s}, 10 \mathrm{~m} / \mathrm{s}$ and $33 \mathrm{~m} / \mathrm{s})$ which represent the minimum, intermediate and maximum flow velocity in the cavitation tunnel. All three analyses were carried out considering only 2D hydrofoil geometry and k- $\varepsilon$ realizable turbulence model. This combination provides a good accuracy of results and lower computational time. All simulations were computed using ANSYS Fluent 18.2.

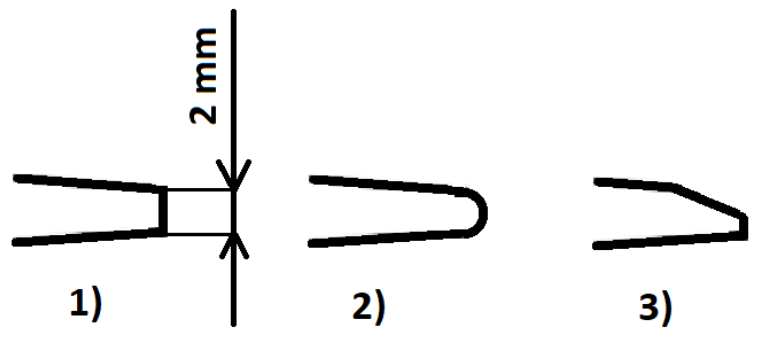

Fig. 2: Trailing edge shapes

\subsection{Computation setup}

The dimensions of the fluid domain correspond to the cavitation tunnel test section (Figure 2). In all three cases, the mesh was created using ca. 140000 quadrilateral cells which provide better convergence and higher accuracy of results. For all simulations the value of $\mathrm{Y}+$ on the hydrofoil surface was checked to ensure that $\mathrm{Y}+$ values lie between the range 20 and 150 as recommended for using wall functions. However, to keep the value of $\mathrm{Y}+$ in this range for the inlet velocity of $2 \mathrm{~m} / \mathrm{s}$ the coarser mesh was created including ca. 85000 quadrilateral cells. At the inlet of the domain, three values of flow velocity were prescribed. According to the trailing edge thickness, the value of hydraulic diameter was set up to $0,002 \mathrm{~m}$. At the outlet of the domain, zero static gauge pressure was applied. At the sides of the domain, free slip boundary condition was set up. The hydrofoil surface was considered as a wall with no-slip boundary condition was applied. Normally, the number of discrete points per oscillation must be higher than 40. Taking into account a Karman vortex shedding frequency of about $3000 \mathrm{~Hz}$ (flow velocity $33 \mathrm{~m} / \mathrm{s}$ ), the time step $5 \mathrm{e}-6 \mathrm{~s}$ was chosen, providing 66 discrete points per oscillation. 


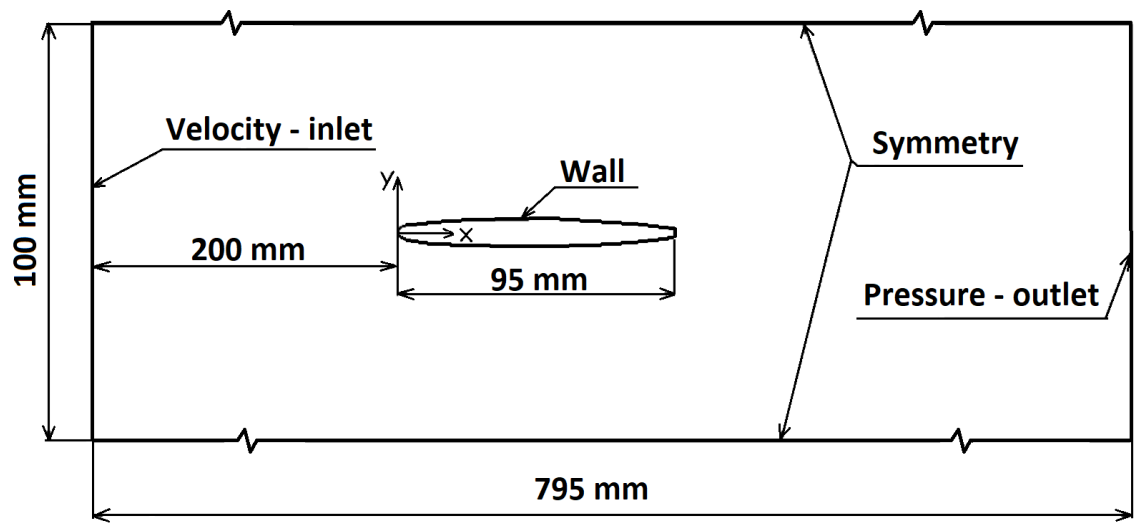

Fig. 3: Fluid domain

\subsection{Transient results}

The unsteady simulation requires a steady state solution as initial condition. This was obtained performing enough iterations to reach the convergence with residuals lower than 1e-06. To observe fully developed vortex shedding, 10000 time steps were computed. However, in some cases additional time steps had to be added. The flow velocity in y-direction and pressure were monitored in 6 points downstream of the hydrofoil (Figure 3). In addition, the lift and drag coefficients $C_{L}$ and $C_{D}$ were evaluated to determine the lift and drag force acting on the hydrofoil. The vortex shedding frequency was calculated using fast Fourier transform of the $C_{L}$ signal (Table 1). For flow velocity of $2 \mathrm{~m} / \mathrm{s}$ no Karman vortex street was observed in the computational model. The oscillations of $\mathrm{C}_{\mathrm{L}}$ coefficient for flow velocity $10 \mathrm{~m} / \mathrm{s}$ were damped due to the trailing edge shape of hydrofoil 3 and therefore no vortex shedding was observed for this case.

Tab. 1: Vortex shedding frequency overview

\begin{tabular}{cccc}
\hline Flow velocity & Hydrofoil 1 & Hydrofoil 2 & Hydrofoil 3 \\
\hline $2 \mathrm{~m} / \mathrm{s}$ & - & - & - \\
\hline $10 \mathrm{~m} / \mathrm{s}$ & $848.9 \mathrm{~Hz}$ & $944.5 \mathrm{~Hz}$ & - \\
\hline $33 \mathrm{~m} / \mathrm{s}$ & $3113.5 \mathrm{~Hz}$ & $3360.6 \mathrm{~Hz}$ & $4168.9 \mathrm{~Hz}$ \\
\hline
\end{tabular}

\section{Modal analysis of the blades submerged in water}

Considering the hydrofoil as an elastic body during the experiments, two regimes of vortex shedding may appear, depending on the vortex shedding frequency and hydrofoil oscillations frequency. If the vortex shedding frequency is in phase with the hydrofoil oscillations, i.e. the resonance is present, the lock-in regime can be observed (Ausoni et al., 2006). If the resonance is not present, the vortex shedding in lockoff regime is observed. To ensure that the lock-in regime can be established, the natural frequencies of all three hydrofoils both in air and still water were determined.

\subsection{Modal analysis of hydrofoils in air}

The first six natural frequencies of all three hydrofoils were computed. The mesh was created using total of 2840 quadratic hexahedral elements (14 200 nodes). The zero values of displacement in $\mathrm{x}, \mathrm{y}$ and $\mathrm{z}$ direction were applied on both hydrofoil sides. This corresponds to the mounting of the hydrofoil in the cavitation tunnel test section. For results overview see Table 2. 
Tab. 2: Hydrofoils natural frequencies in air and in water

\begin{tabular}{ccccccc}
\hline & \multicolumn{2}{c}{ Hydrofoil 1 } & \multicolumn{2}{c}{ Hydrofoil 2 } & \multicolumn{2}{c}{ Hydrofoil 3 } \\
\hline Mode & Air & Water & Air & Water & Air & Water \\
\hline 1 & $1547.2 \mathrm{~Hz}$ & $950.9 \mathrm{~Hz}$ & $1547.8 \mathrm{~Hz}$ & $952.4 \mathrm{~Hz}$ & $1550.3 \mathrm{~Hz}$ & $954.6 \mathrm{~Hz}$ \\
\hline 2 & $2253.9 \mathrm{~Hz}$ & $1553.0 \mathrm{~Hz}$ & $2255.7 \mathrm{~Hz}$ & $1553.0 \mathrm{~Hz}$ & $2261.4 \mathrm{~Hz}$ & $1553.0 \mathrm{~Hz}$ \\
\hline 3 & $3820.3 \mathrm{~Hz}$ & $1716.9 \mathrm{~Hz}$ & $3824.6 \mathrm{~Hz}$ & $1719.3 \mathrm{~Hz}$ & $3839.5 \mathrm{~Hz}$ & $1723.6 \mathrm{~Hz}$ \\
\hline 4 & $5073.1 \mathrm{~Hz}$ & $2663.2 \mathrm{~Hz}$ & $5075.8 \mathrm{~Hz}$ & $2669.0 \mathrm{~Hz}$ & $5084.3 \mathrm{~Hz}$ & $2678.2 \mathrm{~Hz}$ \\
\hline 5 & $6025.5 \mathrm{~Hz}$ & $3039.6 \mathrm{~Hz}$ & $6039.4 \mathrm{~Hz}$ & $3039.6 \mathrm{~Hz}$ & $6081.8 \mathrm{~Hz}$ & $3039.6 \mathrm{~Hz}$ \\
\hline 6 & $6539.8 \mathrm{~Hz}$ & $3965.1 \mathrm{~Hz}$ & $6550.3 \mathrm{~Hz}$ & $3969.0 \mathrm{~Hz}$ & $6589.8 \mathrm{~Hz}$ & $3976.1 \mathrm{~Hz}$ \\
\hline
\end{tabular}

\subsection{Hydrofoils submerged in water}

The dynamics of oscillating hydrofoil submerged in water is strongly affected by added effects which come from the fluid. In this case the added mass effect plays the dominant role and causes the shift of hydrofoil natural frequencies. For proper calculation of submerged hydrofoil natural frequencies the water in the test section was included into the modal analysis. The fluid domain which corresponds to the test section dimensions was represented by 32740 hexahedral acoustic elements (total of 152000 nodes). The hydrofoil was fully fixed on its both sides as in previous case and the FSI Interface was set up on the hydrofoil surface which was in contact with the water. Then the first six natural frequencies were calculated (Table 2). It is obvious, that due to the presence of the water the natural frequencies decrease about $31-55 \%$.

\section{Conclusions}

The new cavitation tunnel has been designed at Viktor Kaplan Department of Fluid Engineering BUT. The preliminary CFD tests were carried out to determine the vortex shedding frequency for three hydrofoils with various trailing edge shapes. Modal analysis of all three hydrofoils submerged in water was carried out, to determine if the vortex shedding in lock-in regime may be observed. Based on the results, the first natural frequency of hydrofoil 1 and hydrofoil 2 lies in the range between vortex shedding frequencies for flow velocity $10 \mathrm{~m} / \mathrm{s}$ and $33 \mathrm{~m} / \mathrm{s}$. This means that the lock-in regime can be established during the future experiments.

The cavitation tunnel will be used for studying the effect of trailing edge shape on wake dynamics. Apart from this, flow induced vibrations and added effects acting from the fluid on the elastic hydrofoil will be studied.

\section{Acknowledgement}

This work has been supported by the grant no. TH02020705 "Research of turbine blade oscillations with respect to extended regulation range for guarantee of stability and safety of energetic system" of the Technology Agency of the Czech Republic and specific research project no. FSI-S-17-4615 "Multiphysics Problems in Fluid Mechanics".

\section{References}

Ausoni, P., et al. (2006) Kármán vortex shedding in the wake of a 2D hydrofoil: Measurement and numerical simulation. In: IAHR International Meeting of the Workgroup on Cavitation and Dynamic Problems in Hydraulic Machinery and Systems. Barcelona, Spain

Dörfler, P., Sick, M. and Coutu, A. (2013) Flow-induced pulsation and vibration in hydroelectric machinery: engineer's guidebook for planning, design and troubleshooting. New York: Springer, ISBN 978-1447142515.

Lockey, K.J., et al. (2006) Flow-induced vibrations at stay vanes: experience on site and CFD simulations. Hydropower \& Dams, issue 5. pp. 102-106

Nedyalkov, I. (2012) Design of Contraction, Test Section, and Diffuser for a High-Speed Water Tunnel. Master thesis. Chalmers University of Technology, Gothenburg. 